\title{
CYCLOTELLA OCELLATA PANTOCSEK (BACILLARIOPHYCEAE): PRIMERA CITA EN CHILE Y COMENTARIOS SOBRE SU VARIABILIDAD MORFOLOGICA
}

\section{CYCLOTELLA OCELLATA PANTOCSEK (BACILLARIOPHYCEAE): FIRST REPORT IN CHILE AND COMMENTS ON ITS MORPHOLOGICAL VARIABILITY}

\author{
Patricio Rivera $^{1}$, Fabiola Cruces $^{1} \&$ Irma Vila $^{2}$ \\ ${ }^{1}$ Departamento de Botánica, Universidad de Concepción, Casilla 160-C, Concepción, \\ Chile. E-mail: privera@udec.cl \\ ${ }^{2}$ Laboratorio de Limnología, Departamento de Ciencias Ecológicas, Universidad de Chile, \\ Casilla 653, Santiago, Chile. E-mail: limnolog@abello.dic.uchile.cl
}

\section{RESUMEN}

Cyclotella ocellata Pantocsek es señalada por primera vez para las aguas continentales de Chile, encontrada en una

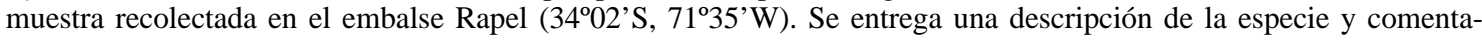
rios relacionados con su variabilidad morfológica.

Palabras Claves: Cyclotella ocellata, diatomeas, Rapel, Stephanodiscaceae.

ABSTRACT

Cyclotella ocellata Pantocsek is reported for the first time from the Chilean freshwaters, found in a sample collected in Rapel reservoir $\left(34^{\circ} 02^{\prime} \mathrm{S}, 71^{\circ} 35^{\prime} \mathrm{W}\right)$. A description of the species and comments on its morphological variability are given.

KEYwords: Cyclotella ocellata, diatoms, Rapel, Stephanodiscaceae.

\section{INTRODUCCION}

Las especies del género Cyclotella (Kützing) Brébisson son muy comunes en los ambientes dulceacuícolas de todo el planeta, aunque algunas se presentan también en aguas salobres, e incluso en ambientes marinos costeros (Hustedt 1930; Lange \& Syvertsen 1989). Cyclotella se caracteriza por presentar sobre la cara valvar un área central con diferente morfología a la existente en la zona marginal. Las diferencias que presenta con los restantes géneros de la familia Stephanodiscaceae Makarova (Stephanodiscus
Ehrenberg, Cyclostephanos Round y Puncticulata Håkansson) fueron recientemente muy bien descritas e ilustradas por Håkansson (2002).

Los representantes de estos géneros están sujetos a una gran variación morfológica, lo que a menudo origina problemas en su determinación específica (Inokuchi \& Maruyama 1990; Håkansson \& Kling 1990; Economou-Amilli 1991; Teubner 1995; Kiss et al. 1996; Håkansson 2002). Recientemente, Rivera et al. (2003) y Tapia et al. (2003) citaron por primera vez para Chile a especies de los géneros Stephanodiscus y Cyclostephanos respectivamente, encontradas 
en material proveniente del lago Chungará y de otros lagos altoandinos.

Hasta la fecha, nueve taxones de Cyclotella han sido señalados para las aguas chilenas, siempre con una abundancia relativa muy baja. Ellos son C. caspia Grunow, C. astraea (Ehrenberg) Kützing (como Stephanodiscus astraea (Ehr.) Grunow), C. glomerata Bachmann, C. kuetzingiana Thwaites, C. meneghiniana Kützing, C. operculata (Agardh) Kützing (=C. distinguenda Hustedt), C. planctonica Brunnthaler, C. stelligera (Cleve \& Grunow) van Heurck y C. stelligera var. major Rivera. Cyclotella comta (Ehr.) Kützing también señalada para Chile es actualmente Puncticulata comta (Ehr.) Håkansson (Håkansson 2002).

El análisis con microscopía fotónica de muestras fitoplanctónicas recolectadas durante el año 2000 en el embalse Rapel, Chile central, evidenció la presencia, en una de ellas, de una diatomea céntrica con forma de tambor. Las observaciones posteriores llevadas a cabo utilizando técnicas de microscopía electrónica permitieron reconocer a Cyclotella ocellata Pantocsek, especie aún no señalada para el país. Este es un taxón que, a pesar de tener una amplia distribución geográfica en ambientes dulceacuícolas, no ha sido profusamente señalado. En Sudamérica sólo existen citas para Argentina (MartínezMacchiavello \& Salas-Aramburu 1994) y Uruguaya, (arroyo Parao, Santibañez 1939, como $C$. kuetzingiana var. planetophora). Barrett et al. (1997) también lo encontraron en el monte Feather, Antárctica.

En el presente trabajo se entrega una descripción de Cyclotella ocellata, comentarios relacionados con su morfología y variabilidad, y diversas fotomicrografías ilustran sus principales características.

\section{MATERIALES Y METODOS}

La presente investigación se realizó sobre la base de una muestra recolectada en el embalse Rapel, ubicado en Chile central (34\%10'S; $71^{\circ} 29^{\prime} \mathrm{W}$ ), a $140 \mathrm{~km}$ al SW de la ciudad de Santiago. Este lago, situado aproximadamente a $70 \mathrm{~m}$ sobre el nivel del mar, tiene una profundidad máxima de $90 \mathrm{~m}$ y un volumen de $0,832 \mathrm{~km}^{3}$ (Cabrera-Silva
1979), y es un cuerpo de agua que presenta valores de $\mathrm{pH}$ que varían entre 8 y 9 , y una temperatura entre 7 y $25^{\circ}$ (Vila et al. 2000). Ha sido definido como un lago eutrófico con altos niveles de nutrientes ( $\mathrm{P}$ y $\mathrm{N}$ total), debido principalmente a las actividades turísticas y agrícolas desarrolladas en su cuenca (Vila et al. 1997). Además, sus sedimentos contienen altos niveles de metano y sulfatos como consecuencia de los desechos provenientes de la minería del cobre de la región (Vila et al. 2000).

La muestra analizada fue colectada el 21 de junio de 2000 en el sector Muro del embalse mediante una botella Van Dorn, y fijada con solución de lugol. Este material se encuentra depositado en la Colección Diatomológica de la Universidad de Concepción bajo el número DIAT-CONC M-3206 (DIAT-CONC 6458, 6459, 6460). Se eliminó la materia orgánica de los frústulos siguiendo el método descrito por Hasle \& Fryxel (1970), y el material fue montado finalmente en Hyrax. Los microscopios utilizados fueron un Fotomicroscopio III Zeiss con condensador de contraste de fases, un ETEC Autoscan U-1 (microscopio electrónico de barrido) y un JEOL JEM-1200 EX (microscopio electrónico de transmisión), los dos últimos pertenecientes al Laboratorio de Microscopía Electrónica de la Universidad de Concepción, Chile. La terminología adoptada en este trabajo corresponde a la propuesta por Ross et al. (1979) y por Håkansson (2002).

\section{RESULTADOS}

\section{DESCRIPCIÓN}

Cyclotella ocellata Pantocsek, p. 104, Lám. 15, Fig. 318 (1901). Figs. 1-26

Sinonimia: Cyclotella crucigera Pantocsek, p. 104, Lám. 15, Fig. 325 (1901). Cyclotella tibetana Hustedt, p. 117, Fig. 19 (1922).

Referencias: Håkansson, p. 266, Figs. 11-17, 42-44 (1990). Håkansson in Krammer \& Lange-Bertalot, p. 51, Lám. 50, Figs.1-11, 13 14; Lám. 51, Figs. 1-5 (1991). Håkansson, p. 310, Figs. 1-8 (1993). Håkansson, p. 85, Figs. 309-318 (2002). 

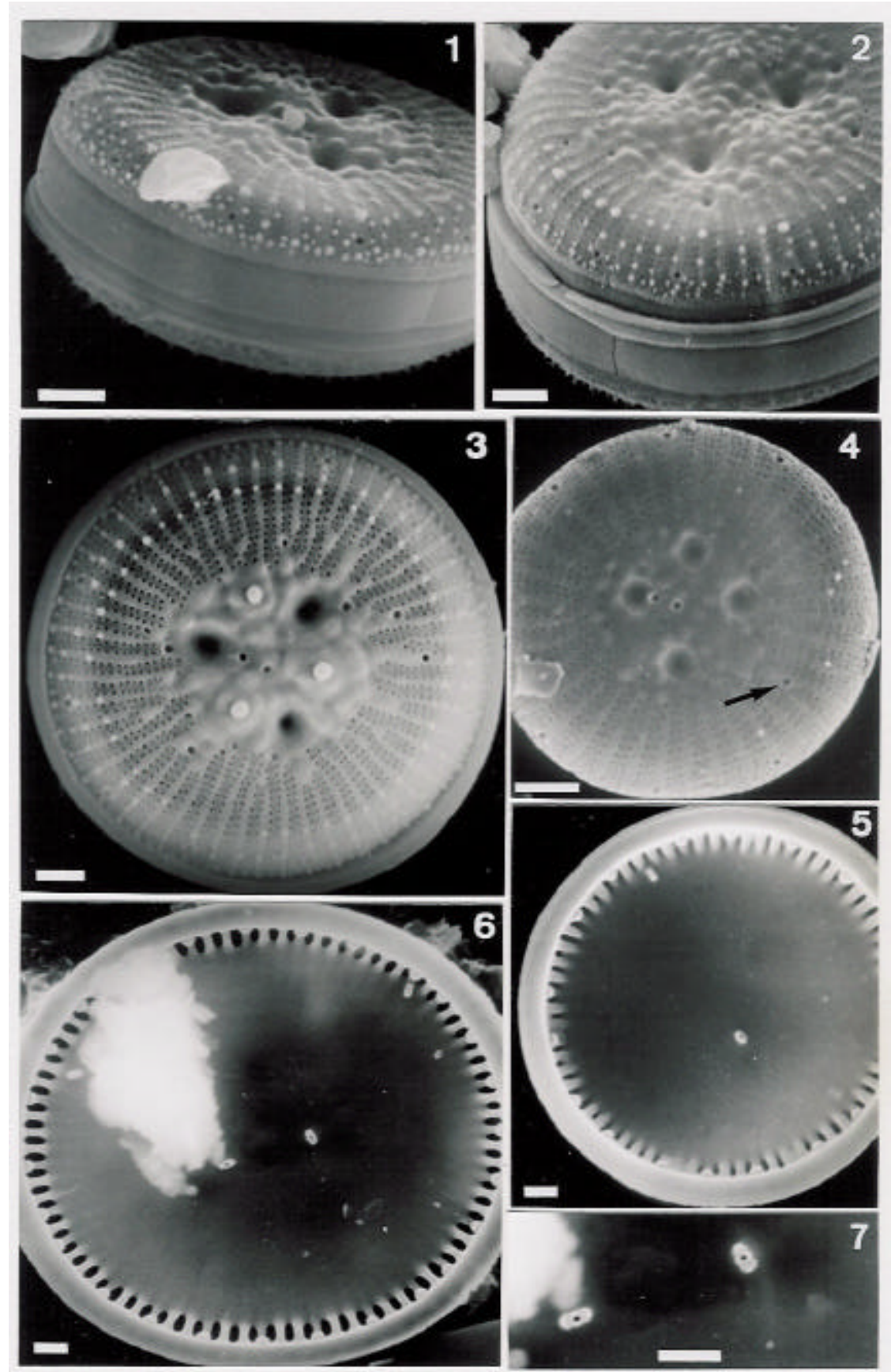

Figuras 1-7. Cyclotella ocellata. SEM. Escala $=1 \mu \mathrm{m}$. Frústulo rectangular en vista conectival; cingulum formado por bandas no perforadas; valvas casi planas con gránulos de sílice sobre el manto. Fig. 2. Epicingulum formado por tres bandas, valvocópula más ancha; valva con tres depresiones y tres papilas en el área central. Fig. 3. Tres depresiones y tres papilas en el área central; estrías formadas por una doble línea de perforaciones; algunas interestrías están bifurcadas. Fig. 4. Cuatro depresiones sobre la cara valvar; interestrías no bifurcadas; la flecha indica la abertura externa del proceso labiado. Fig. 5. Vista interna de una valva, lisa, con un proceso reforzado valvar y un proceso labiado orientado radialmente. Fig. 6. Vista interna de valva con dos procesos reforzados valvares y dos procesos labiados orientados en forma oblicua. Fig. 7. Vista interna de procesos reforzados valvares, cada uno con dos poros satélites. SEM: microscopía electrónica de barrido. 


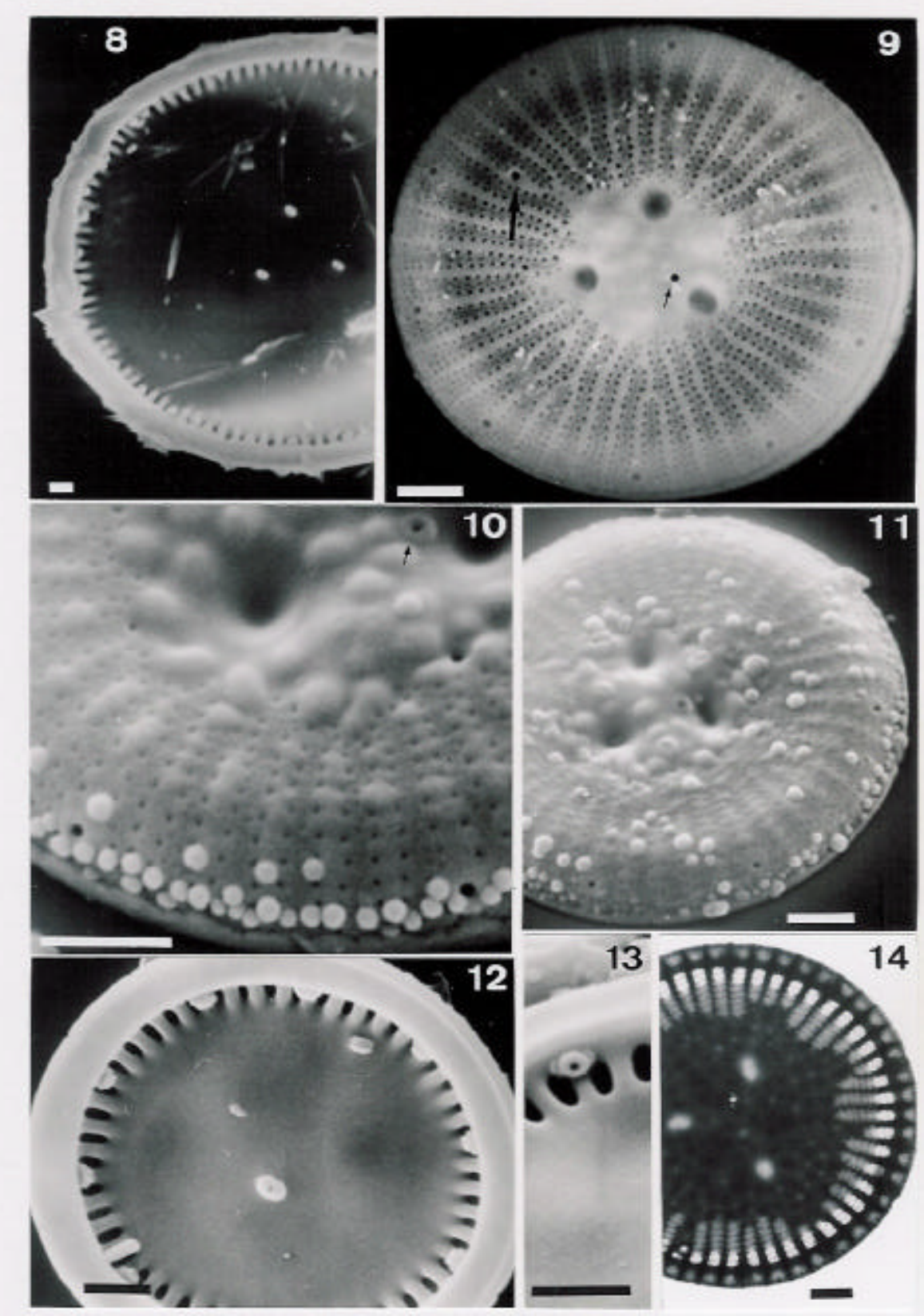

Figuras 8-14. Cyclotella ocellata. Figs. 8-13 SEM, Fig. 14 TEM. Escala $=1 \mu \mathrm{m}$. Fig. 8. Vista interna de una valva con tres procesos reforzados valvares y dos procesos labiados. Fig. 9. Vista externa de una valva con tres depresiones en una pequeña área central; la flecha más pequeña señala la abertura externa de un proceso reforzado valvar, y la más grande la de un proceso labiado; entre las interestrías, algunas bifurcadas, se encuentran 3-4 líneas de pequeñas perforaciones. Fig. 10. Area central externa coliculada; la flecha indica la abertura externa de un proceso reforzado central; estrías marginales formadas por dos líneas de perforaciones. Fig. 11. Pequeños granos de sílice se ubican incluso sobre la cara valvar. Fig. 12. Vista valvar interna con un proceso reforzado valvar y un proceso labiado. Fig. 13. Proceso reforzado marginal provisto de dos poros satélites. Fig. 14. Valva con área central grande y estrías marginales no divididas. $\mathrm{SEM}=$ microscopía electrónica de barrido. TEM= microscopía electrónica de transmisión. 
Células solitarias, rectangulares en vista conectival, con forma de tambor (Fig. 1). Cingulum formado por hasta tres bandas abiertas, no perforadas, siendo la valvocópula mucho más ancha que las pleuras (Figs. 1-2). Valvas circulares, casi planas, 4,8-26,8 $\mu \mathrm{m}$ de diámetro, divididas en dos zonas concéntricas: una zona central carente de aréolas y por una zona marginal provista de estrías (Figs. 3-4, 9, 14-23). Area central 2,1$10,8 \mu \mathrm{m}$ de diámetro, longitud que corresponde a un $30-70 \%$ del diámetro valvar. Superficie exterior central provista de pequeñas elevaciones (Figs. 1$3,10-11$ ), pero lisa interiormente (Figs. 5-8, 12). Normalmente existen tres papilas sobre la cara exterior del área central (Figs. 2-3, 9, 11, 15-23), raramente cuatro, acompañadas de sus respectivas depresiones (Fig. 4). Los procesos reforzados de la cara valvar se ubican en la zona subcentral, cada uno provisto de dos poros satélites (Figs. 7, 12) y con la abertura externa circular, algo engrosada (Figs. 9-10, flechas pequeñas). En las valvas de diámetro reducido existe sólo un proceso reforzado (Figs. 5, 9, 12, 14), pero hasta tres en las de mayor tamaño (Fig. 8). Estrías marginales con estructura alveolada simple, 14-18(22) en $10 \mu \mathrm{m}$, con poca diferencia de longitud en las valvas pequeñas (Figs. 15-18), la cual se acentúa notablemente en las de mayor diámetro (Figs. 19-23), estructuradas exteriormente por una doble línea de pequeñas aréolas opuestas entre sí (Figs. 3-4, 10), aumentando a tres o cuatro líneas cerca del margen en algunas valvas (Fig. 9); interiormente presentan una pared de sílice lisa que deja una abertura alargada (Figs. 5-6, 8, 12). Interestrías levemente elevadas sobre la superficie valvar (Figs. 1-2, 10). En las células de pequeño tamaño, tanto las estrías como las interestrías continúan directamente hasta el margen valvar (Figs. 4, 14-16), pero en las valvas de mayor diámetro algunas interestrías se ramifican dicotómicamente (Figs. 3, 9, 17-23). Sobre las interestrías del manto valvar se sitúan numerosos gránulos de sílice (Figs. 1-3, 10), y excepcionalmente también sobre toda la cara valvar (Fig. 11). Un anillo de procesos reforzados se encuentra en el manto valvar (Figs. 5-6, 8, 12), algo más cerca del margen que de la unión cara valvar/manto (Figs. 2-4, 9-11). Estos procesos, separados cada 4-6 (27) interestrías, presentan una abertura externa de contorno circular (Figs. 9-10) y un tubo interno corto y grueso, provisto de dos poros satélites orien- tados en dirección circular (Fig. 13). Un proceso labiado (Figs. 5, 12), a veces dos (Figs. 6, 8), se ubica sobre la cara valvar, relativamente cerca de la unión con el manto valvar; su abertura externa es circular (Figs. 4, 9, flechas grandes) e internamente es sésil y el labium se orienta generalmente en forma oblicua respecto al radio de la célula (Figs. $6,8,12)$.

\section{Microflora ACOMPAÑANTE}

En el material estudiado en el presente trabajo, $C$. ocellata se presentó en forma relativamente abundante, acompañada de Aulacoseira granulata (Ehr.) Simonsen, Cyclotella meneghiniana Kützing, Asterionella formosa Hassall, Diatoma tenuis Agardh, Hannaea arcus (Ehr.) Patrick, Synedra ulna (Nitzsch) Ehrenberg, Cocconeis placentula Ehr. var. euglypta (Ehr.) Cleve, Cymbella minuta Hilse, Cymatopleura solea (Bréb.) Wm. Smith, Nitzschia dissipata (Kütz.) Grunow, Stephanodiscus kuetzingii Klee \& Casper y diversas especies de Clorofíceas.

\section{DISCUSION}

De acuerdo con los criterios morfológicos que permiten diferenciar las especies (Håkansson 1996, 2002), nuestra investigación demuestra que el material analizado corresponde a Cyclotella ocellata Pantocsek, taxón que no había sido señalado anteriormente para Chile (Tabla I).

Sobre la base de las observaciones realizadas en más de un centenar de valvas del material chileno, se comprueba, una vez más, la variabilidad de algunos caracteres morfológicos de esta especie. El labium del proceso labiado se orienta en la mayoría de los individuos en forma oblicua al radio de la célula, pero en algunos de ellos tuvo una orientación claramente radial (Fig. 5). Kiss et al. (1996) lo observaron incluso orientado en ángulo recto con respecto al radio de la valva. Los procesos reforzados del manto valvar se ubican generalmente cada 4-6 interestrías, tal como lo describe Håkansson (1991, 1993 y 2002), pero en algunos individuos del material chileno éstos se encontraban cada dos interestrías (Fig. 6), e incluso hasta cada siete (Fig. 11), concordando con lo observado por Kiss et al. (loc. cit.) Es importante co- 
Gayana Bot. 60(2), 2003

mentar aquí que, aunque sin mencionarlo, Håkansson (1990) en su figura 45 muestra una valva de $C$. ocellata recolectada en Suecia, donde los procesos reforzados del manto están separados cada 2-4 interestrías. También varía el número de inte- restrías en el margen valvar que, señalado para la especie como de 13-15 en $10 \mu \mathrm{m}$, fluctuó entre 14 y 22 (14-23 en Kiss et al., loc. cit.), aunque la mayor parte de las valvas presentaron entre 14-18 en $10 \mu \mathrm{m}$.

TABLA I. Comparación de las características morfológicas de Cyclotella ocellata encontradas en el presente estudio con las descritas por otros autores.

\begin{tabular}{|c|c|c|c|c|}
\hline & Presente estudio & $\begin{array}{c}\text { Håkansson 1990, } 1993 \\
\text { (material tipo) }\end{array}$ & Kiss etal., 1996 & Håkansson 2002 \\
\hline $\begin{array}{l}\text { Diámetro }(\mu \mathrm{m}) \text { y } \\
\text { superficie valvar }\end{array}$ & 4,8-26,8; casi plana & --;plana & 4,9-27,3; casi plana & 8-25; casi plana \\
\hline $\begin{array}{l}\mathrm{N}^{\mathrm{o}} \text { depresiones y } \\
\text { papilas en el centro }\end{array}$ & $3-4$ & $3-4$ & $3-5$ & 3-5 (omás) \\
\hline $\begin{array}{l}N^{o} \text { procesos reforzados } \\
\text { y poros satélites valva }\end{array}$ & $1-3 / 2$ & $1-2 / 2$ & $1-6 / 2(0-1)$ & $1-2 / 2$ \\
\hline $\begin{array}{l}\text { Posición procesos } \\
\text { reforzados y poros } \\
\text { satélites en el manto }\end{array}$ & cada 2-7 interestrías $/ 2$ & $\begin{array}{c}\text { cada5-6 } \\
\text { interestrías } * / 2\end{array}$ & $\begin{array}{c}\text { cada 1-6 } \\
\text { interestrías / } 2\end{array}$ & cada 3-6/2 \\
\hline $\begin{array}{l}\text { Largo estrías } \\
\text { área central }\end{array}$ & $\begin{array}{l}\text { algo imegulares en valvas } \\
\text { pequeñas y muy diferentes } \\
\text { en las de mayor tamaño }\end{array}$ & - & imegulares & algoimegulares \\
\hline $\begin{array}{l}\text { Estrías continuas o } \\
\text { bifurcadas en manto }\end{array}$ & $\begin{array}{l}\text { continuas en valvas } \\
\text { pequeñas y bifurcadas } \\
\text { en las de mayor tamaño }\end{array}$ & continuas* & bifurcadas & continuas \\
\hline $\begin{array}{l}\mathrm{N}^{\mathrm{o}} \text { interestrías en el } \\
\text { margen }(10 \mu \mathrm{m})\end{array}$ & $14-22$ & - & $14-23$ & - \\
\hline $\begin{array}{l}\mathrm{N}^{\mathrm{o}} \text { proceso (s) labiado } \\
\text { (s); estructura y } \\
\text { orientación }\end{array}$ & 1-2; sésil, oblicuo o radial & 1; oblicuo & $\begin{array}{l}\text { 1-3; oblicuo, radial o } \\
\text { enángulo recto }\end{array}$ & al menos 1; sésil, oblicuo \\
\hline
\end{tabular}

\footnotetext{
* ver mayor información en el texto.

— sin información
} 


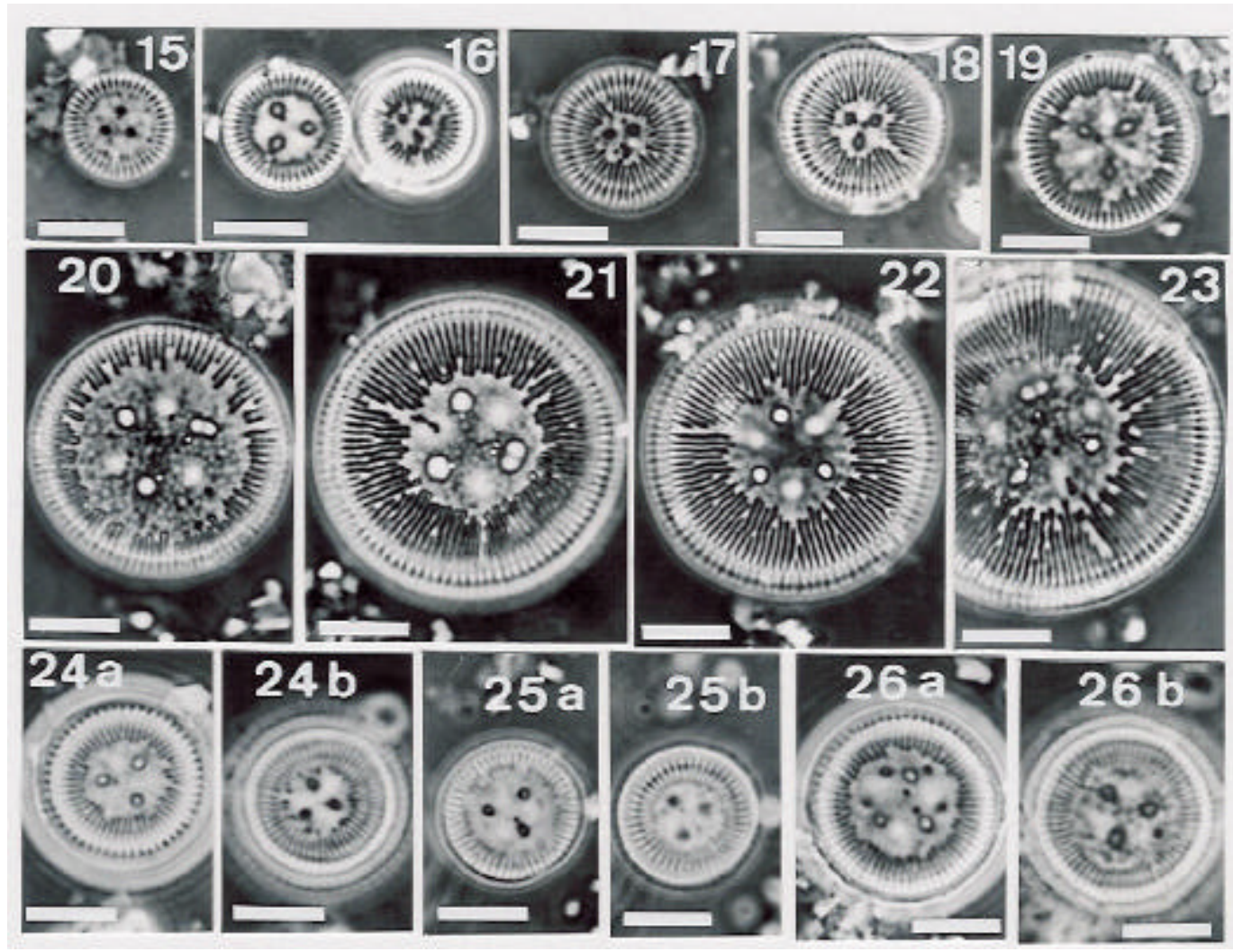

Figs. 15-26. Cyclotella ocellata. LM. Escala $=5 \mu \mathrm{m}$. Resumen de la variabilidad morfológica de la especie con relación al tamaño del área central, largo de las estrías marginales y bifurcación de las interestrías. Figs. 24-25. Se muestran las dos tecas $(\mathrm{a}+\mathrm{b})$ de frústulos que poseen áreas centrales de diferente tamaño. Fig. 26. Las áreas centrales de las dos valvas de un mismo frústulo $(\mathrm{a}+\mathrm{b})$ no parecen diferir significativamente en tamaño. LM= microscopía fotónica.

El diámetro del área central de las valvas es un carácter muy variable, situación que no parece tener relación con el tamaño de los individuos (Figs. 15-26). Algunas figuras de Håkansson 1990, 1993 (material tipo) y 1991 muestran áreas centrales de diferentes tamaños. En el material chileno encontramos frústulos donde su tamaño en la epivalva es muy diferente al de la hipovalva (Figs. 24-25), característica que no parece presentarse en todos los individuos (Fig. 26). Teubner (1995) estudió material de $C$. ocellata recolectado en lagos y lagunas de Berlín, y encontró que en muchas células ambas valvas de un mismo frústulo presentaban diferentes características morfológicas. Las figuras 11a y $11 \mathrm{~b}$ de esta autora muestran valvas con el patrón de "C. ocellata", donde una de ellas presenta un área central de tamaño notablemente mayor que la existente en la valva opuesta. Kiss et al. (1996) y
Hegewald \& Hindakova (1997) también afirmaron que los individuos de esta especie son heterovalvares. Kiss et al. (1999), encontraron una gran variabilidad morfológica en esta especie, incluso en el material tipo de C. ocellata.

El número de procesos reforzados valvares, de procesos labiados y la presencia de interestrías bifurcadas se relaciona con el tamaño de los individuos. Las células de tamaño reducido presentan siempre un solo proceso reforzado valvar, pero en muchas valvas que superan los $10 \mu \mathrm{m}$ de diámetro existen dos o tres. Una situación similar ocurre con el proceso labiado, donde hasta dos de ellos fueron observados en valvas mayores de $15 \mu \mathrm{m}$ de diámetro. En el material del lago Las Madres, Kiss et al. (loc. cit.) encontraron hasta seis procesos reforzados valvares y hasta tres procesos labiados, cuyo número estaba directamente relacionado con 
el tamaño del diámetro valvar. Con respecto a las interestrías del área marginal, se ha descrito como característico de $C$. ocellata que ellas continúan sin interrupción hasta el margen de la valva (Håkansson 1993, 2002). Sin embargo, en nuestro material esto ocurre sólo en las valvas de diámetro pequeño, pues en frústulos más grandes $(9-10 \mu \mathrm{m})$ se presentan algunas interestrías bifurcadas, característica que se hace constante para todos los individuos aún más grandes. Aunque tampoco comentado por Håkansson (1990), la valva de $C$. ocellata ilustrada en su figura 45 también presenta varias interestrías bifurcadas dicotómicamente. Lo mismo se observa en las Figs. 6 y 8 (lám. 50) y Fig. 1 (lám. 51) de Håkansson 1991, y en el material de Kiss etal.(loc. cit.) las valvas siempre presentaron interestrías divididas.

Algunos caracteres morfológicos son menos variables para la especie. Estos son la estructura del cingulum y del área central, la ubicación y número de poros satélites de los procesos reforzados valvares y del manto, y la ubicación y estructura interna de los procesos labiados.

Debido al polimorfismo valvar existente en C. ocellata y en otras especies afines, que muchas veces no permite llevar a cabo una certera determinación específica, diversos autores han considerado algunas de ellas como sinónimos de las otras. CleveEuler (1951) considera a C. ocellata como sinónimo de C. kuetzingiana var. planetophora Fricke. La variabilidad valvar de representantes del género Cyclotella observada por Teubner (1995) en los lagos de Alemania la llevan a considerar que $C$. krammeri Håkansson (=C. kuetzingiana Thwaites), $C$. kuetzingiana var. planetophora Fricke, $C$. kuetzingiana var. radiosa Fricke, C. ocellata Pantocsek y $C$. comensis Grunow corresponden a una misma especie. Hegewald \& Hindakova (1997), al estudiar material de una población natural de C.ocellata recolectado cerca de Colonia-Alemania, observaron una gran variabilidad morfológica (especialmente referida al número, forma y orientacion de las depresiones valvares, número de los procesos labiados y orientación del labiuminterno, número y distribución de los procesos reforzados valvares y del manto, entre otros), y concluyeron que las células de gran tamaño son similares a Cyclotella krammeri (=C. kuetzingiana Thwaites), mientrasqueotras son idénticas a $C$. rossii Håkansson. Por el momento, los únicos sinónimos de C.ocellata que parecen estar definitivamente aceptados por los diatomólogos son $C$. crucigera Pantocsek y C.tibetana Hustedt.
Con excepción de Stephanodiscus kuetzingii Klee \& Casper, que recientemente encontramos en Rapel, todas las especies de diatomeas que acompañaban a $C$. ocellata en la muestra estudiada fueron anteriormente señaladas para este embalse (Cabrera et al. 1977, Montecino \& Cabrera 1982, Vila et al. 1987), y son taxones comunes para las aguas continentales de Chile (Rivera 1983, Rivera et al. 1990). Vila et al. (loc. cit.) señalaron la presencia en Rapel de C. meneghiniana Kützing, pero el dibujo correspondiente a este taxón (Fig. 2-1) es erróneo puesto que representa una valva con la ornamentación marginal como la de esta especie, pero su área central corresponde a la de C.ocellata.

\section{AGRADECIMIENTOS}

Los autores agradecen a la Dra. Nora I. Maidana (Argentina), Dra. Carina Lange y Dr. Roberto Rodríguez (Chile), y a un revisor anónimo, por sus valiosas sugerencias y comentarios al manuscrito. Igualmente agradecen la colaboración de los señores Raúl Alarcón, Hugo Pacheco y Julio Pugin, personal del Laboratorio de Microscopía Electrónica de la Universidad de Concepción.

\section{BIBLIOGRAFIA}

Barrett, P.J., N.L. Bleakley, W.W. Dickinson, M.J.Hannah \& M.A. HARPER. 1997. Distribution of siliceous microfossils on Mount Feather, Antarctica, and the age of the Sirius Group. En: The Antarctic Region: Geological Evolution and Processes (ed. C.A. Ricci), pp. 763-770. Terra Antártica Publication, Siena, Italy.

Cabrera-Silva, S. 1979. La clorofila "a" como indicador de la biomasa presente en dos estuarios y un lago de Chile Central. Publicación Ocasional, Museo Nacional de Historia Natural, Santiago 29: 3-10.

Cabrera S., V. Montecino, I. Vila, N. Bahamonde, I. Bahamondes, I. Barends, R. Rodríguez, R. Ruiz \& D. Sото. 1977. Características limnológicas del embalse Rapel, Chile central. Publicación de O.E.A. sobre el Seminario de Medio Ambiente y Represas, Montevideo, Uruguay. Monografía Departamento de Asuntos Científicos y Tecnológicos 1: 40-61.

Cleve-Euler, A. 1951. Die Diatomeen von Schweden und Finnland. Kungliga Svenska Vetenskaps-Akademiens Handlingar $4^{\text {th }}$. Ser., Band 2(1), 163 pp.

Economou-Amilli, A. 1991. Cyclotella elymaea, a new fossil species from the Neogene Basin of Kozani- 
Aeani-Servia, Northern Greece. Diatom Research 6: $223-233$

HÅKANSSON, H. 1990. A comparison of Cyclotella krammeri nov. sp., $C$. rossii nov. sp. and C. schumanni nov. stat. with similar species. Diatom Research 5: 261271.

HÅkansson, H. 1991. Cyclotella, Cyclostephanos, Stephanodiscus, Thalassiosira, Stephanocostis. En: Bacillariophyceae. Süsswasserflora von Mitteleuropa, Band 2/3, 3. Teil. (eds. K. Krammer \& H. Lange-Bertalot), pp. 40-81. G. Fischer, Stuttgart \& New York.

HÅkAnsson, H. 1993. Morphological and taxonomic problems in four Cyclotella species (Bacillariophyceae). Diatom Research 8: 309-316.

HÅKAnsson, H. 1996. Cyclotella striata complex: typifications and new combinations. Diatom Research 11: 241-260.

HÅKansson, H. 2002. A compilation and evaluation of species in the general Stephanodiscus, Cyclostephanos and Cyclotella with a new genus in the family Stephanodiscaceae. Diatom Research 17: 1-139.

HÅkansson, H. \& H. KuING. 1990. The current status of some very small freshwater diatoms of the genera Stephanodiscus and Cyclostephanos. Diatom Research 5: 273-287.

Hasle, G.R. \& G.A. Fryxell. 1970. Diatoms: Cleaning and mounting for light and electron microscopy. Transactions of the American Microscopical Society 89: 469-474.

Hegewald, E. \& A. Hindakova. 1997. Variabilität von einer natürlichen Population und von Klonen des Cyclotella-ocellata-Komplexes (Bacillariophyceae) aus dem Gallbergweier, Nordwestdeutschland. Algological Studies 86: 17-37.

Hustedt, F. 1922. Bacillariales aus Innerasien, gesammelt von Dr. Sven Hedin. En: Southern Tibet, Band VI, Teil III, Bot. (ed. S. Hedin), pp.107-152, 2 Taf.

Hustedt,F. 1930. Die Kieselalgen Deutschland, Österreichs und der Schweiz. L. Rabenhorst's "KryptogamenFlora von Deutschland, Österreich und der Schweiz". 7: 1-920, 542 Abb.

Inokuchi, M. \& K. Maruyama. 1990. Infraspecific differences in Cyclotella comta populations in the Fuji Lakes and Lake Ashino-ko in Japan. Japanese Journal of Phycology 38: 105-117.

Kiss, K.T., R. KleE \& E. Hegewald. 1999. Reinvestigation of the original material of Cyclotella ocellata Pantocsek (Bacillariophyceae). Algological Studies 93: 39-53.

Kiss, K.T., C. Rojo \& M.A. Cobelas. 1996. Morphological variability of a Cyclotella ocellata (Bacillariophyceae) population in the Lake Las Madres (Spain). Algological Studies 82: 37-55.

LANGe, C. \& E.E. SyverTSEn. 1989. Cyclotella litoralis sp. nov.
(Bacillariophyceae), and its relationships to $C$. striata and $C$. stylorum. Nova Hedwigia 48: 341-356.

Martínez-Macchiavello, J.C. \& W. Salas-Aramburu. 1994. Catálogo Taxonómico-ecológico de las diatomeas no-marinas recientes y fósiles de la República Argentina. Instituto Antártico Argentino, Buenos Aires. Publicación No 27, 113 pp.

Montecino, V. \& S. Cabrera. 1982. Phytoplankton activity and standing crop in an impoundment of central Chile. Journal of Plankton Research 4: 943-950.

Pantocsek, J. 1901. A Balaton Kovamoszatai vagy Bacillariái, 17 Pls., Budapest. 143 pp.

RIVERA, P. 1983. A Guide for References and Distribution for the Class Bacillariophyceae in Chile between $18^{\circ} 28^{\prime} \mathrm{S}$ and $58^{\circ} \mathrm{S}$. Bibliotheca Diatomologica, Vol. 3, $386 \mathrm{pp}$.

Rivera, P., M. Gebauer \& H.L. Barrales. 1989 (1990). A Guide for References and Distribution for the Class Bacillariophyceae in Chile between $18^{\circ} 28^{\prime} \mathrm{S}$ and $58^{\circ}$ S. Part II. Data from 1982 to 1988. Gayana Botanica 46:155-198.

Rivera, P., F. Cruces \& I. Vila. (2002) 2003. Primera cita de Stephanodiscus agassizensis Hakansson \& Kling (Bacillariophyceae) en Chile. Gayana Botanica 59: 79-86.

Ross, R., E.J. Cox, N.I. Karayeva, D.G. Mann, T.B.B. Paddock, R. Simonsen \& P.A. Sims. 1979. An Amended Terminology for the Siliceous Components of the Diatom Cell. Nova Hedwigia Beihefte 64: 513-533.

SANTIBAÑEZ, I. 1939. Contribución al conocimiento de las diatomeas uruguayas. Revista Sudamericana de Botánica 6: 6-9.

TAPIA, P.M., E.C. Theriot, S.C. Fritz, F. CRuces \& P. RiveRA. 2003. Distribution and morphometric analysis of Cyclostephanos andinus comb. nov., a planktonic diatom from the Central Andes. Diatom Research (en prensa).

Teubner, K. 1995. A light microscopical investigation and multivariate statistical analyses of heterovalvar cells of Cyclotella-species (Bacillariophyceae) from lakes of the Berlin-Brandenburg region. Diatom Research 10: 191-205.

Vila, I., I. Barends \& V. Montecino. 1987. Abundancia y distribución temporal del fitoplancton en el embalse Rapel, Chile central. Revista Chilena de Historia Natural 60: 37-55.

Vila, I., M. Contreras \& J. Pizarro. 1997. Eutrophication and phytoplankton selective responses in a temperate reservoir. Verhandlungen der internationalen Vereinigung für theorethische und andewandte Limnologie 26: 798-802.

Vila, I., M. Contreras, V. Montecino, J. Pizarro \& D. Adams. 2000. Rapel: A 30 years temperate reservoir. Eutrophication or contamination? Archiv für Hydrobiologie 55: 31-44. 